\title{
SYSTEM ANALYSIS AND DESIGN FOR A BUSINESS DEVELOPMENT MANAGEMENT SYSTEM BASED ON SAUDI ARABIA MARKET
}

\author{
Osama S Islam, Bassam Zafar, Ahmed Mueen, Mohammed A. Balubaid, \\ Hani S. Brdesee
}

King Abdulaziz University, Jeddah, Saudi Arabia

\begin{abstract}
A design of a sales system for professional services requires a comprehensive understanding of the dynamics of sale cycles and how key knowledge for completing sales is managed. This research describes a design model of a business development (sales) system for professional service firms based on the Saudi Arabian commercial market, which takes into account the new advances in technology while preserving unique or cultural practices that are an important part of the Saudi Arabian commercial market. The design model has combined a number of key technologies, such as cloud computing and mobility, as an integral part of the proposed system. An adaptive development process has also been used in implementing the proposed design model.
\end{abstract}

\section{KEYWORDS}

Management System, Saudi Arabia market, Cloud computing, Mobility, Adaptive development process

\section{INTRODUCTION}

Small organizations in Saudi Arabia does not currently utilize any specific system or a particular approach for their business development activities, but rather use a number of stand-alone productivity applications such as Microsoft Outlook (for managing appointments / contacts), Microsoft PowerPoint / Word (for templates standardization), Microsoft Excel (for tracking the progress.

Large Cooperative organizations in Saudi Arabia are using Microsoft Dynamics CRM [11] or SAP as a platform for their business development activities i.e. collaboration between employees and contractors, managing contracts, appointments, contacts etc. and our survey of these large cooperative organizations shows that these organizations are fully satisfied by the current approach. However, using Microsoft Dynamics CRM or SAP are over-adequate for small organizations because these organizations require limited features for their business development activities. Furthermore, the cost / maintenance / infrastructure requirement of Microsoft Dynamics CRM or SAP is not feasible for small organizations $[2,3,6]$.

In comparison with the current approach of business development available for small organizations, the proposed Business Development Management System has a wide number of DOI:10.5121/ijfcst.2017.7601 
International Journal in Foundations of Computer Science \& Technology (IJFCST) Vol.7, No.5/6, November 2017

improvements such as standardizing how proposal are developed and followed-up, accuracy of relevant information about opportunities etc. In particular, the proposed Business Development Management System will mainly contribute as a new approach by providing the capabilities of collecting / managing information about potential business opportunities and tracking information about service requests. In addition, business development personnel with access to all of the relevant information needed while they are in the field.

The paper is organized as follows. In Section II, market analysis with reference to Saudi Arabia is presented. The proposed Business Development Management System for Small Organizations is discussed in section III. Discussion, Conclusion and Future work are presented in section IV.

\section{MARKET ANALYSIS}

In general, the field of business development for professional services [1,4,5,13], which includes IT consulting and services, usually have non-transactional steps prior to obtaining a request for proposal. For example, Pollack (2012) in [8] described business development as "the creation of long-term value for an organization from customers, markets, and relationships". This study highlights the strategic role that business opportunities play in creating long-term value for an organization.

Information systems that can support business development objectives are usually bundled within Customer Relationship Management (CRM) systems that cover the following major processes in the marketing and sales cycle:

- Contact Management, to track and manage key information on relevant individuals related to opportunities and client organizations.

- Lead Management, to track and identify potential opportunities and work on transferring them into actual opportunities.

- Opportunity Management, to track and monitor the

progress of deals and proposals.

On the other hand, the currently available CRM systems in the market have grown considerably in the last decade and become very complex, difficult to manage without proper support and specialized personnel to setup / configure. This has presented a major drawback to small or medium organizations that want to leverage such systems but don't want or have the needed investment to setup the required system infrastructure. Further analysis of related information system has identified three major trends, namely on-premises enterprise systems, cloud-based systems and specialized solutions.

\subsection{On-Premises Enterprise Systems:}

The traditional setup for most large organizations is to establish an integrated system for client relationship management that provides a comprehensive look at the whole process of managing the customer life cycles. In addition, these systems have traditionally been deployed by organizations as part of a company-wide business process improvement initiative, which require compressive investment in hardware, software, consulting services and training. For example, 
International Journal in Foundations of Computer Science \& Technology (IJFCST) Vol.7, No.5/6, November 2017

major vendors such as Microsoft, Oracle have provided a number of CRM product lines to serve this sector. In particular Microsoft Dynamics CRM (MSDCRM, 2015) [9] has been wellpositioned with midsized to large organizations to support sales, service, marketing, social business processes.

\section{Cloud-Based Systems:}

Recent trends in cloud computing has resulted in the emergence of CRM Software As A Service (SaaS) that simplify the deployment of such systems by allowing organizations to focus on the configuration of the system rather than setup and installation. For example, Sales Force (SF, 2015) [10] has progressively provided online cloud services in sales, service, data, marketing and analytics since 1999 and has a leader position in cloud-based CRM. In addition, Microsoft Dynamics CRM Online has also emerged as an online version of its main product to serve the growing cloud market. In addition, most cloud based CRM providers have also created dynamic platforms that can easily be expanded with custom solutions depending upon customer requirements. On the other hand, security is still a main concern for most organizations dealing with cloud-services and continues to be a drawback for its usage.

\section{Specialized Solutions:}

Certain vendors have developed highly specialized solutions that support a mobile workforce. For example, mviSLM developed by Sales Lead Tracking Software (2015, SLTS) [11] provides a solution that runs on mobile devices that allows those is the field to quickly access their data while not in the office.

\section{PROPOSED SOLUTION}

The business development or sales process for professional services is usually unstructured but strategic activity requires flexibility when dealing with new opportunities even following a target plan. The main issue which sales personnel face is that the sale cycle can take months to materialize; however, when multiple leads are followed some does mature before others, which requires a system to track and follow up on each lead. One approach to resolve this issue is to have a Web or Mobile based information system that is accessible to sales personnel while in the field. 
International Journal in Foundations of Computer Science \& Technology (IJFCST) Vol.7, No.5/6, November 2017

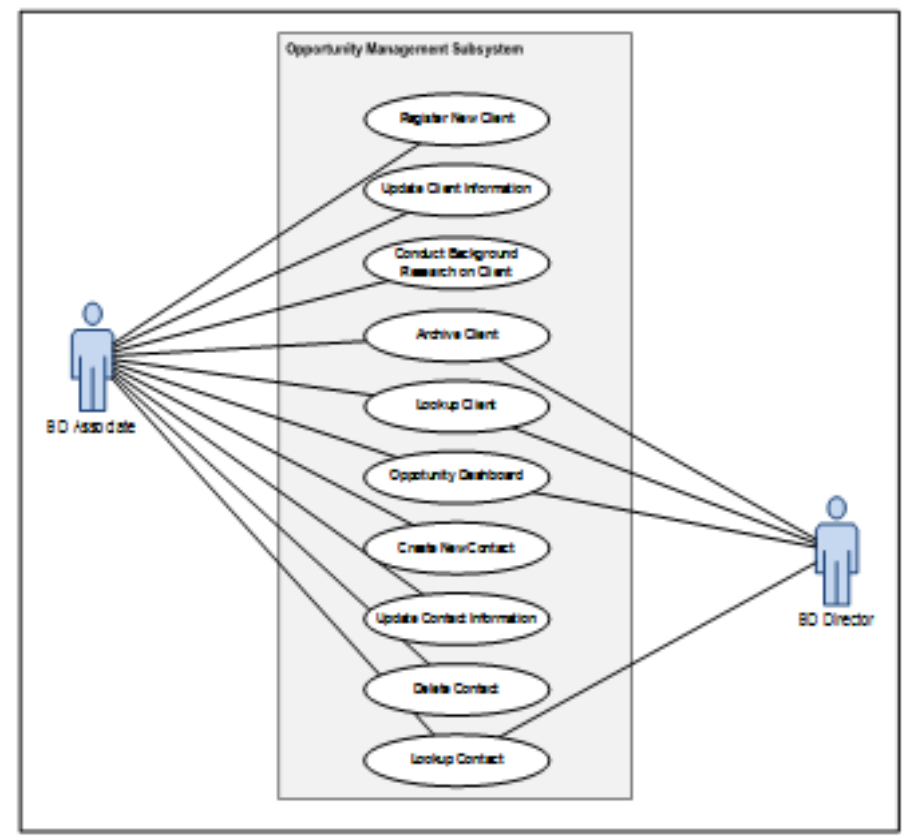

Figure 1: Opportunity Management Subsystem

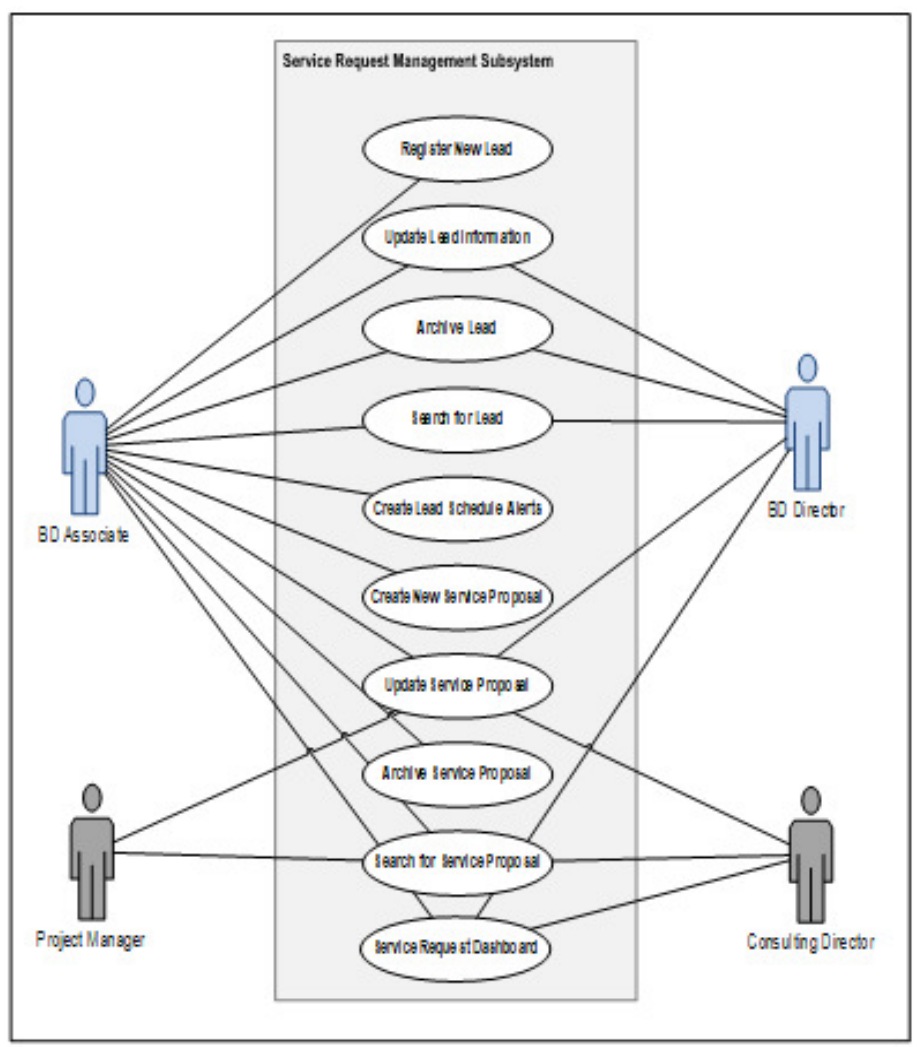

Figure 2: Service Request Management Subsystem 
International Journal in Foundations of Computer Science \& Technology (IJFCST) Vol.7, No.5/6, November 2017

To ensure that the proposed system meets small organization requirements the following functional and non-functional requirements have been identified [7, 12].

\subsection{Functional Requirement}

The figure 1 and 2 shows the relevant use cases and users/actors for each of the two main subsystems of BDMS namely Opportunity Management Subsystem and Service Request Management Subsystem.

\subsection{Non-Functional Requirement}

To ensure that the proposed system meets the organization's operational standards the following non-functional requirements have been identified.

Usability Requirements

- The system ensures more interactivity on the client side.

- A mobile friendly version of the web application with well-designed user interface is required.

\section{Reliability Requirements}

- The system should support scalability without requiring extensive changes.

- The system should provide a local caching backup option that a user can activate to quickly recovery from loss.

- Minimize the number of faults in the system to below $2 \%$ on average.

- The client side modules of the system should be able to operate in offline mode in case of network connection failure and resynchronize once connection is reestablished.

- The system should backup critical information in the database and a transaction log of all daily operations is maintained to ensure minimum loss of information.

Performance Requirements

- The client side module of the system should be responsive to user requests within 1-3 seconds.

- System uptime should be above $98 \%$, and should be fault tolerant.

Security Requirements

- The new system should implement multi-level security functionality that allows only authorized users using registered computers and smartphones to access the system. 
International Journal in Foundations of Computer Science \& Technology (IJFCST) Vol.7, No.5/6, November 2017

In addition, the following expanded set of non-functional requirements has also been identified.

Design Constraints

- The client side module for desktop users should work with major browsers available in the market.

Implementation Requirements

- The system should be based on modular approach.

Physical Requirements

- The server infrastructure will be deployed in a cloud service Platform As A Service (PaaS) to enable easier management and scalability of infrastructure if needed.

Supportability Requirements

- The server side installation should not disrupt the current daily operation of the business.

- The system maintenance needs to be performed either during non-working hours or during the weekends.

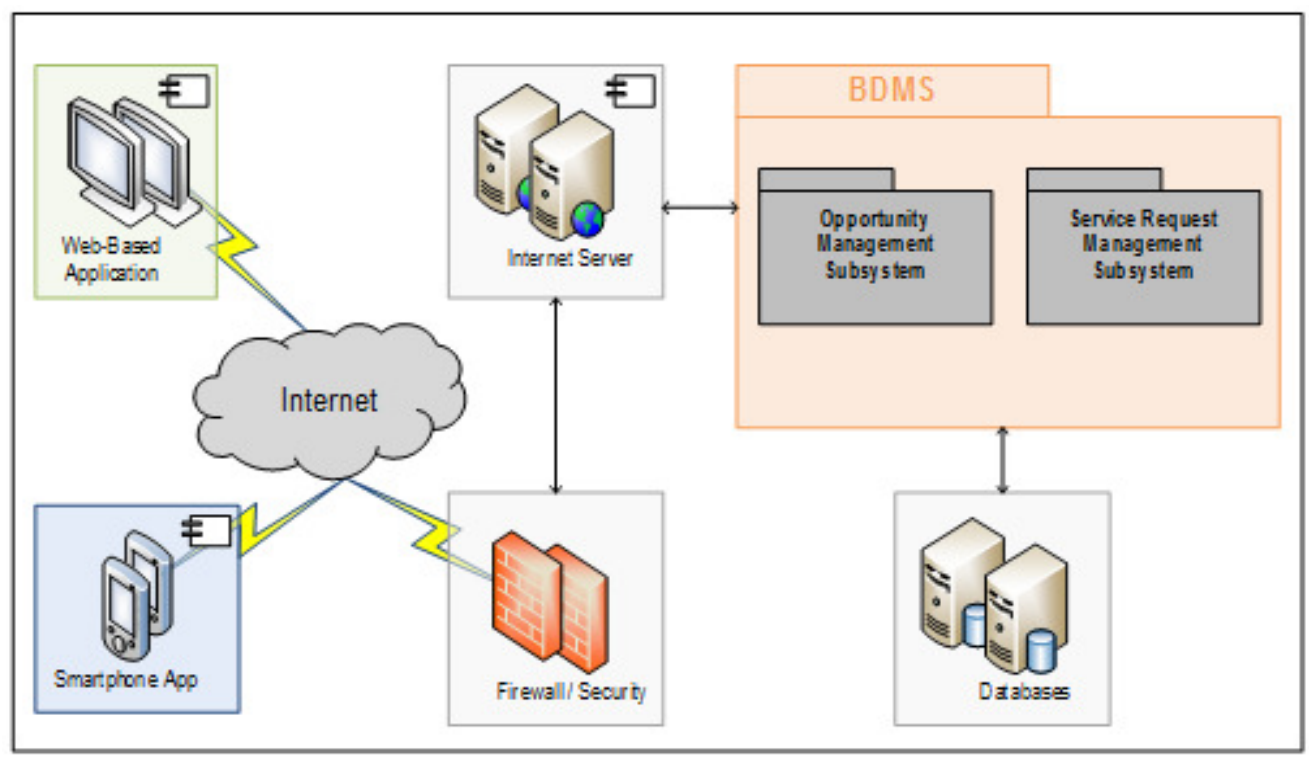

Figure 3: High-Level Architecture of Proposed System 
International Journal in Foundations of Computer Science \& Technology (IJFCST) Vol.7, No.5/6, November 2017

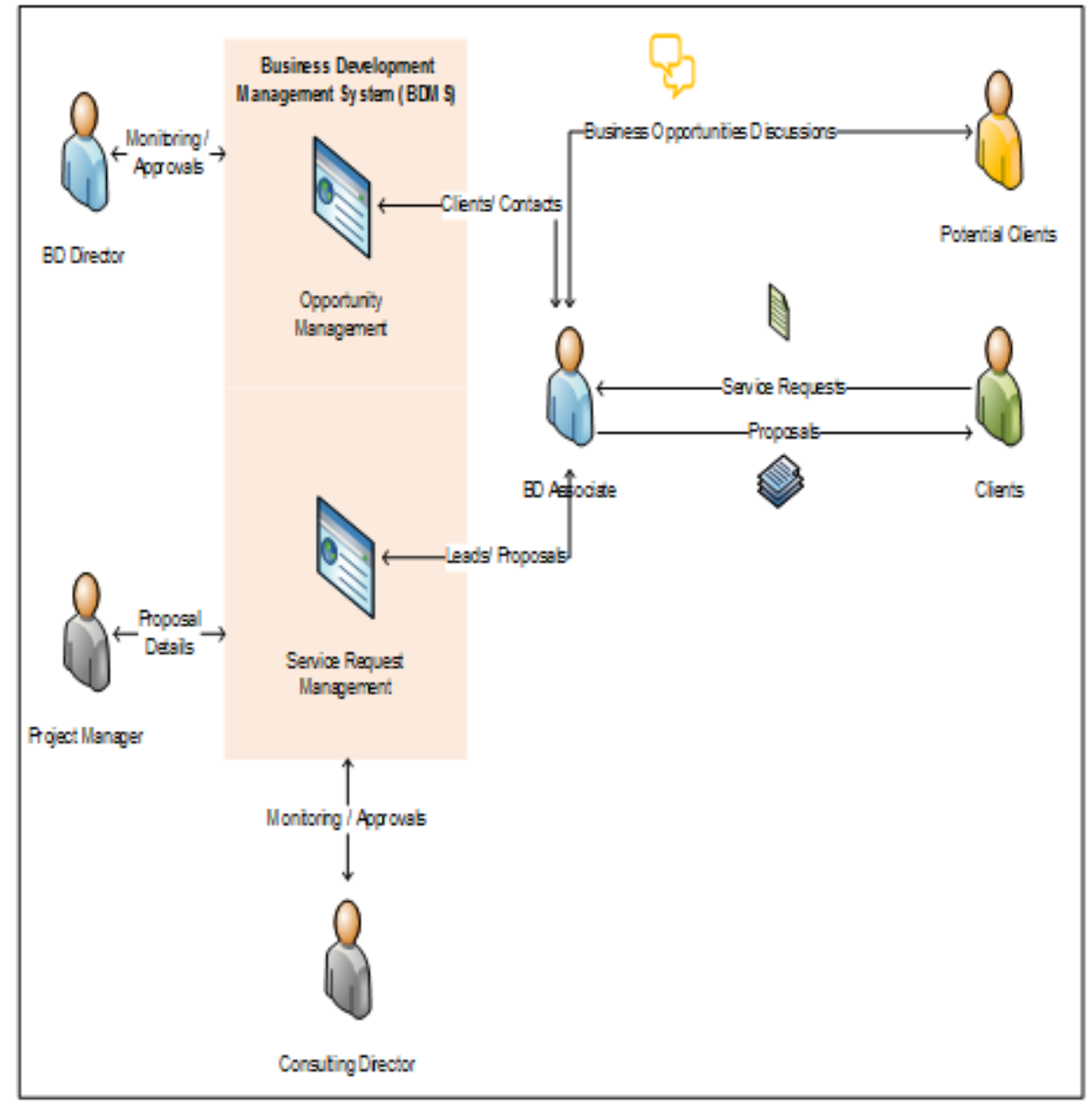

Figure 4: Workflow of Proposed System

\subsection{System Architecture}

The figure 3 shows the high-level system architecture of the proposed system with subsystems and modules and figure 4 shows the workflow of the proposed system. Opportunity Management Subsystem is responsible for managing the opportunity, follow-up and monitoring to ensure that all relevant information / contact details are available to transform an opportunity to a business lead.

Service Request Management Subsystem is responsible for handling a lead, starting form a simple service request to providing a full proposal / quotation. Figure 5 shows the System architecture whereas figure 6 shows the Layered architecture of the proposed system respectively.

Opportunity Management interacts with the BD Associate and BD Director with the main goal of transforming an opportunity to a lead. In addition, using this module the BD Associate can better communicate with the potential clients to discuss potential business opportunities. Service 
International Journal in Foundations of Computer Science \& Technology (IJFCST) Vol.7, No.5/6, November 2017

Request Management interacts with BD Associate, Project Manager, and Consulting Director with the main goal of submitting a proposal to the client.

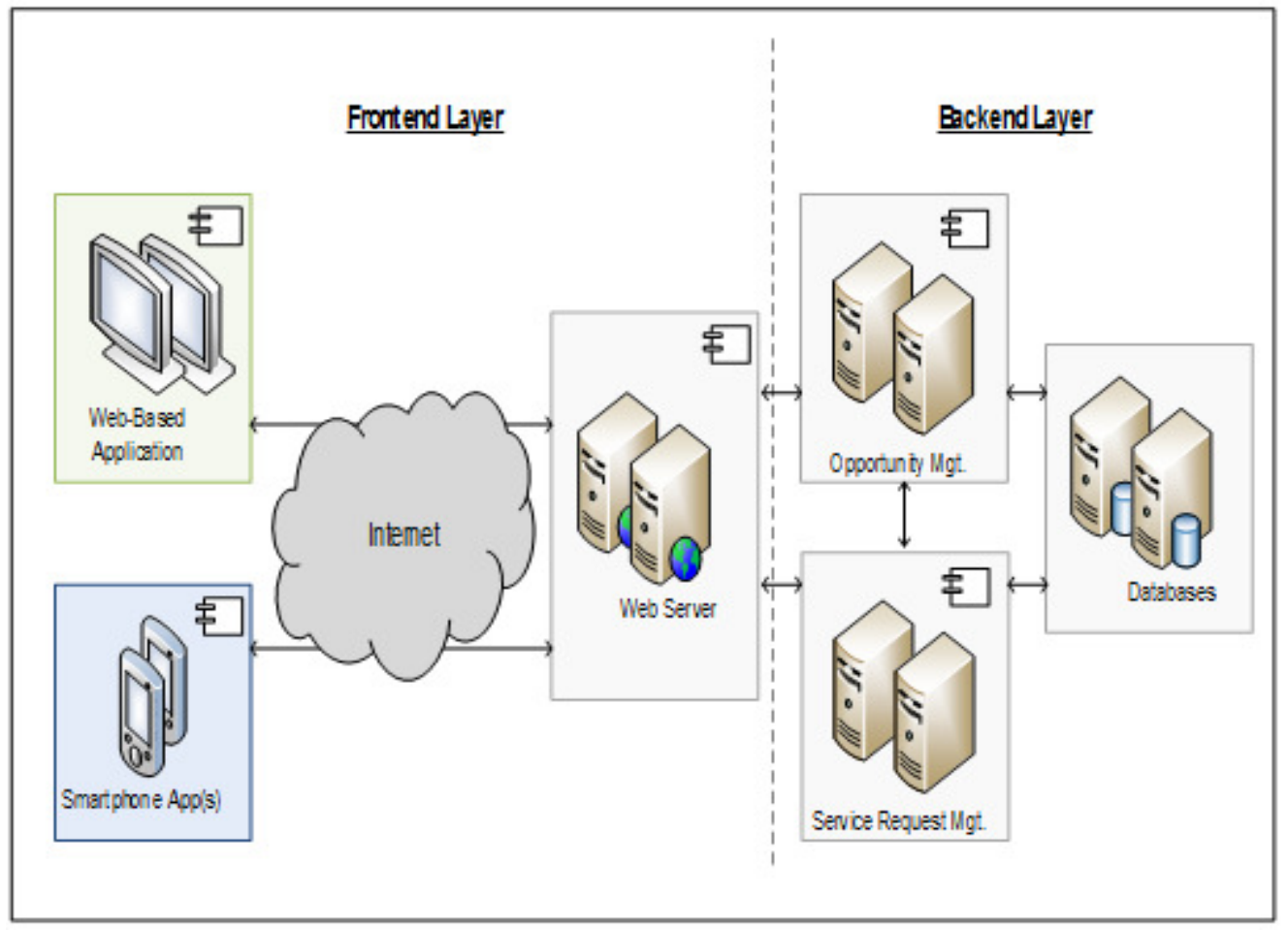

Figure 5: System Architecture of Proposed System

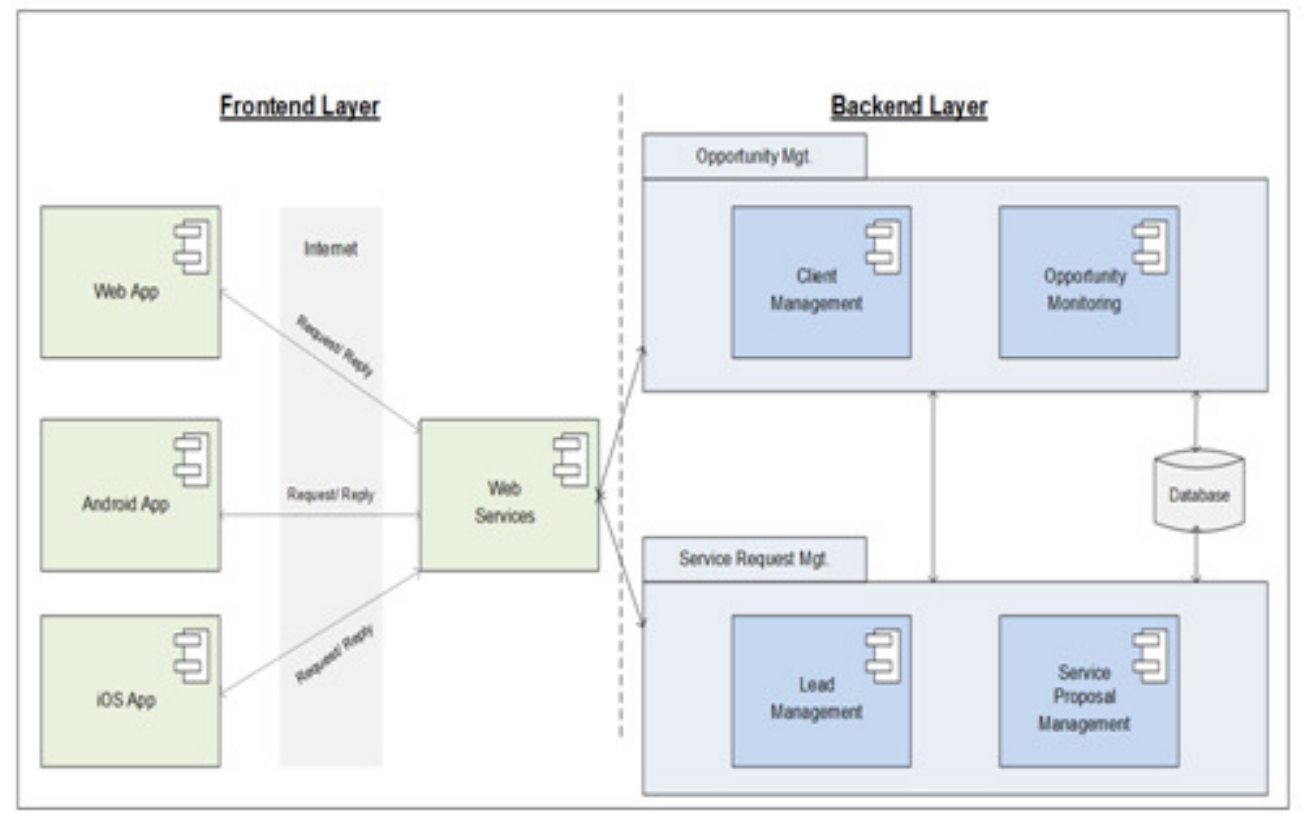

Figure 6: Layered Architecture of Proposed System 
The proposed BDMS is enterprise-level system and is developed in Java; mobile app is developed on Android version 4.1 and is compatible with all new versions of Android. The system has been deployed in two small organizations; the evaluation results were satisfactory and support the implementation of the solution.

\section{DISCUSSION AND CONCLUSION}

Based on the evaluation results collected after deploying; the proposed system plays a key role in transforming the local Saudi business into a more efficient and effective organization. These improvements are a result of enhancing the accuracy of opportunity information, standardizing how proposal are developed and how quick they can be delivered to a potential client. At the same time, such a change needs to be done with care as the sales team in the organization must accept the use of the new system and keep it up-to-date with relevant information for such a system to succeed. However, with the support and commitment of the general manger and owner this should assist in achieving the needed transformation. On the other hand, the proposed BDMS system in principle is based on CRM concepts that sales team members should be able to understand and appreciate, however to avoid the complexities, the BDMS is proposed to provide the needed functional and non-functional requirements to the organization. From a design perspective the solution is proposed to be a two-layered web system to simplify how the system is programmed and maintained in the future. The frontend layer handles the user interface and control system components. This provides the flexibility in meeting user interface requirements, either web or device specific, and providing dynamic web services that can scale to meet future needs. The proposed solution is developed using Java to support cross-platform, however, the mobile app is developed on Android and in future can developed for other platforms such as iOS if needed.

\section{REFERENCES}

[1] Lidia Ogiela, Marek R. Ogiela (2014) "Cognitive systems for intelligent business information management in cognitive economy" International Journal of Information Management, Volume 34, Issue 6, Pages 751-760.

[2] Fatemeh Rahimi, Charles Møller, Lars Hvam (2016) "Business process management and IT management: The missing integration" International Journal of Information Management, Volume 36, Issue 1, Pages 142-154.

[3] Peter P. Groumpos (2015) "Modelling Business and Management Systems Using Fuzzy Cognitive Maps: A Critical Overview", IFAC-PapersOnLine, Volume 48, Issue 24, Pages 207-212.

[4] Jan vom Brocke, Sarah Zelt, Theresa Schmiedel (2015) "On the role of context in business process management", International Journal of Information Management, In Press, Available online.

[5] Xinwei Zhu, Seppe vanden Broucke, Guobin Zhu, Jan Vanthienen, Bart Baesens (2016) "Enabling flexible location-aware business process modeling and execution", Decision Support Systems, Volume 83, Pages 1-9.

[6] Mona von Rosing, Henrik von Scheel, Justin Tomlinson, Victor Abele, Kenneth D. Teske, Michael D. Tisdel (2015) "Business Process Management Alignment", The Complete Business Process Handbook, Pages 645-656. 
International Journal in Foundations of Computer Science \& Technology (IJFCST) Vol.7, No.5/6, November 2017

[7] Umar Mansoor, Mohammed Balubaid, Muhammad Usman, Ahmed Mueen (2015) "Ontology-Based Clinical Decision Support System for Predicting High-Risk Pregnant Woman” International Journal of Advanced Computer Science and Applications (IJACSA): Vol 6, No. 12, 203-2088.

[8] Nurussobah Hussin, Rusnah Johare (20154 "Functional requirements for an electronic records management system for court records", Concepts and Advances in Information Knowledge Management, Pages 73-90.

[9] IS (2015) I-SnapShot [Online] (Accessed: 1 April 2015). Available from: http://www.i-snapshot.com.

[10] MSDCRM (2015) Microsoft Dynamics CRM [Online] (Accessed: 1 April 2015) Available from: http://www.microsoft.com/en-us/dynamics/crm.aspx

[11] Pollack, S. (2012) What, Exactly (Accessed: 1 April 2015) Is Business Development? [Online]. Available from: http://www.forbes.com/sites/scottpollack/2012/03/21/what-exactly-is-businessdevelopment/

[12] SF (2015) SalesForce.com [Online] (Accessed: 1 April 2015). Available from: http://www.salesforce.com

[13] SLTS (2015) Mobile Access to Leads With Mobile Sales Software [Online]. Available from: http://www.mvislm.com/mobile_sales_workforce.php 